\title{
About renormalized effective action for the Yang-Mills the- ory in four-dimensional space-time
}

\author{
A.V. Ivanov $^{1, *}$ \\ ${ }^{1}$ St. Petersburg Department of V.A. Steklov Institute of Mathematics of the Russian Academy of Sci- \\ ences, 27 Fontanka, St. Petersburg, Russia
}

\begin{abstract}
This work is related to the asymptotic approach in the renormalization theory and its problems. As the main example, the Yang-Mills theory in four-dimensional space-time is considered. It has been shown earlier [16] that using the asymptotic of the bare coupling constant one can find an expression for the renormalized effective action, however, this formula has problems (divergence $\ln \varepsilon$ and infinite series). This work shows the relation of these values and provides an answer for the renormalized effective action.
\end{abstract}

\section{Introduction and results}

\subsection{Introduction}

The main object of consideration is the effective action for the quantum Yang-Mills theory in four-dimensional space-time in the formalism of the background field $B_{\mu}(x)$ method ([4]-[7]), which can be represented ([8]-[10]) as

$$
W(B, \alpha)=\frac{1}{\alpha} W_{-1}+W_{0}+\sum_{k=1}^{\infty} \alpha^{k} W_{k},
$$

where $\alpha$ is a coupling constant, $W_{-1}=W_{-1}(B)$ is the classical action of the Yang-Mills theory and $W_{n}=W_{n}(B)$ for $n \geqslant 0$ corresponds to the contribution of strongly connected vacuum diagrams with $n+1$ loops.

The traditional procedure of renormalization ([13]-[15]) can be described using a forest formula. This procedure allows to eliminate the divergences, however, it depends on the regularization and renormalization prescriptions. It is very important that the considered example has asymptotic freedom ([1], [2]) and dimensional transmutation [3]. After applying the renormalization procedure with dimensional regularization ([11], [12]), wich is described in section 3.1, the effective action takes the form (23), where $\alpha_{r}$ is the renormalized coupling constant and $W_{n, 0}$ for $n>0$ is a finite part of $W_{n}$.

But it is possible to obtain the renormalized effective action by different way, which is named assymptotic approach to the renormalization and is described in [16]. The main essence of the method is to find the asymptotic behavior of the bare coupling constant $\alpha(\varepsilon)$ and to use the

*e-mail: regul1@mail.ru 
last one for limit transition when $\varepsilon \rightarrow+0$. After some calculations the asymptotic behavior of renormalized effective action can be expanded in series

$$
W_{\text {ren }}(B)=-\frac{\beta_{2}}{\beta_{1}} W_{-1} \ln \varepsilon+(C+\ln (\mu / p)) W_{-1}+W_{1 \text { loop }}(p)+\sum_{k=1}^{\infty}(-1)^{k} \frac{W_{k, k}}{\beta_{1}^{k}}+o(1),
$$

where $p$ is the arbitrary massive parameter, $W_{1 \text { loop }}(p)$ is a finite part of $W_{0}, \beta_{1}$ and $\beta_{2}$ are coefficients of the $\beta$ - function, $W_{k, k}$ for $k>0$ is a coeffitient near $\left(\alpha_{r} / \varepsilon\right)^{k}$ and $C$ is the constant and can be expressed by coefficients of $\beta$ - function.

It is very easy to see that the formula (2) has some problems. First of all it is the logarithmic singularity $\ln \varepsilon$ when $\varepsilon \rightarrow+0$ because theory after renormalization should not has the divergences, moreover the series (1) after regularization does not have singularities like $\ln \varepsilon$. The second problem is related to series with infinity number of terms.

This work is devoted to the problems described above. The theorem, obtained in section 3.2 by using properties of $Z$ - function ([17]) from section 2, gives answer for $W_{k, k}$ for $k>0$. After this result it is possible to solve described problem.

\subsection{Results}

As it is mentioned above the main theorem gives the formulas for coefficients $W_{k, k}$ when $k>0$. First of all it is convenient to consider the fourth term in (2), but nothing is known about the convergence, therefore, it is necessary to cut the series and consider

$\sum_{k=1}^{f(\varepsilon)}(-1)^{k} \frac{W_{k, k}}{\beta_{1}^{k}}=\sum_{k=1}^{f(\varepsilon)}(-1)^{k} \frac{W_{-1}(-1)^{k-1} \beta_{2} \beta_{1}^{k-1}}{\beta_{1}^{k}(k+1)}=-\frac{\beta_{2}}{\beta_{1}} W_{-1} \sum_{k=1}^{f(\varepsilon)} \frac{1}{k+1}=-\frac{\beta_{2}}{\beta_{1}} W_{-1}\left(H_{f(\varepsilon)}-1\right)$,

where $H_{k}$ is $k$-th harmonic number (28) and $f(\varepsilon)$ is a regulator for series which is a function of $\varepsilon$ with the property

$$
\ln \varepsilon+H_{f(\varepsilon)}-1=c_{1}+o(1)
$$

when $\varepsilon \rightarrow+0$, where $c_{1}$ is a constant. Using asymptotic behavior for hurmonic number it can be rewritten like

$$
\varepsilon f(\varepsilon) e^{\gamma-1}=e^{c_{1}}(1+o(1)) .
$$

In this way after substituting (3) and (5) in (2) and limit transition $\varepsilon \rightarrow+0$ renormalized effective action has the form

$$
W_{\text {ren }}(B)=\left(C+c_{1}+\ln (\mu / p)\right) W_{-1}+W_{1 \text { loop }}(p) .
$$

The last formula is indicative and instructive, because it reflects the properties of the theory and renormalization.

\section{Definition and basic properties of $Z$ - function}

As is known, most quantum models contain divergences and renormalization methods are used to obtain a physically and mathematically correct theory. Several different renormalization schemes exist however they have a common feature. It is the introduction of an additional Lagrangian which reduces divergences. In the case of the Yang-Mills theory in the four dimensional space-time with dimensional regularization it can be expressed through the introduction of factor $Z$, which is a constant of renormalization. 


\subsection{Definitions}

There are several formulas for $Z$ - function (series in powers of singularities, exponential formula). In this section the first one is studied. So, the series in powers of singularities $1 / \varepsilon$ and coupling constant $\alpha_{r}$ can be written in such view

$$
Z\left(\alpha_{r}\right)=1+\sum_{k=1}^{\infty} \frac{z_{k}\left(\alpha_{r}\right)}{\epsilon^{k}}=1+\sum_{k=1}^{\infty} \sum_{n=k}^{\infty} \frac{z_{n, k} \alpha_{r}^{n}}{\epsilon^{k}} .
$$

To find the relations between the coefficients of effective action it is necessary to know the ones for $Z^{-1}$ and $Z^{m}$ when $m>0$. For this purpose constructions

$$
Z^{m}\left(\alpha_{r}\right)=\sum_{k=0}^{\infty} \frac{1}{\epsilon^{k}} \sum_{n=0}^{\infty} \alpha_{r}^{n+k} z_{n+k, k}^{(m)}, Z^{-1}\left(\alpha_{r}\right)=\sum_{k=0}^{\infty} \frac{1}{\epsilon^{k}} \sum_{n=0}^{\infty} \alpha_{r}^{n+k} z_{n+k, k}^{(-1)}
$$

can be introduced. The general formulas for $z_{n+k, k}^{(m)}$ and $z_{n+k, k}^{(-1)}$ can be written, but there are not convenient, so the main purpose of this section is to find explicit formulas only for the necessary coefficients $\left(z_{k, k}^{(m)}\right.$ for $k>0$ and $m>0, z_{k, k}^{(-1)}$ and $z_{k+1, k}^{(-1)}$ for $\left.k>0\right)$.

\subsection{Differential equations for $z_{k}\left(\alpha_{r}\right)$ and its solutions}

From the general theory it is known that the relation between bare and renormalized coupling constants exist in such view

$$
\alpha(\epsilon)=\mu^{\epsilon} Z\left(\alpha_{r}\right) \alpha_{r}
$$

where $\mu$ and $\varepsilon$ are parameters of regularization. After substituting (7) in (9) and using differentiation by $\mu$ and Gell-Mann-Low equation

$$
\mu \frac{d}{d \mu} \alpha_{r}=-\epsilon \alpha_{r}+\sum_{i=0}^{\infty} \beta_{i+1} \alpha_{r}^{i+2}=-\epsilon \alpha_{r}+\beta\left(\alpha_{r}\right),
$$

the relation (9) can be decomposed in the system of differential equations with initial conditions

$$
-z_{k}^{\prime}\left(\alpha_{r}\right) \alpha_{r}^{2}+\left(z_{k-1}\left(\alpha_{r}\right)+z_{k-1}\left(\alpha_{r}\right) \alpha_{r}\right) \beta\left(\alpha_{r}\right)=0, z_{k}(0)=0, z_{0}=1, k>0 .
$$

Solutions of the system (11) can be written in the following form

\section{Lemma 1:}

$$
z_{k}\left(\alpha_{r}\right)=\int_{0}^{\alpha_{r}} \frac{\left(z_{k-1}(x)+z_{k-1}^{\prime}(x) x\right) \beta(x)}{x^{2}} d x, k>0 .
$$

\subsection{About $z_{k, k}$ and $z_{k+1, k}$ for $k>0$}

By definition function $z_{k}\left(\alpha_{r}\right)$ when $k>0$ can be expressed like a series in powers of coupling constant

$$
z_{k}\left(\alpha_{r}\right)=z_{k, k} \alpha_{r}^{k}+z_{k+1, k} \alpha_{r}^{k+1}+\ldots,
$$

where only the first and the second terms are taken into account. Using the decomposition and (12) relations between coefficients can be written in such form:

Lemma 2:

$$
\begin{gathered}
z_{k, k}=\beta^{k}, k \geqslant 0, \\
z_{2,1}=\frac{\beta_{2}}{2}, z_{k+2, k+1}=z_{k+1, k} \beta_{1}+z_{k, k} \frac{k+1}{k+2} \beta_{2}, k>0 .
\end{gathered}
$$




\subsection{About $z_{k, k}^{(m)}$ for $k>0$ and $m>0$}

The main aim is to find the answer for coefficients $z_{k, k}^{(m)}$ when $m>0$ and $k>0$. It is well known that $z_{k, k}^{(m)}$ is the coefficient for $\left(\alpha_{r} / \varepsilon\right)^{k}$ and $Z$ - function contains constructions $\alpha_{r}^{n} / \varepsilon^{k}$ only for $n \geqslant k$. In this case $k ! z_{k, k}^{(m)}$ is the k-th Taylor coefficient of function

$$
\sum_{k=0}^{\infty} z_{k, k} x^{k}=\sum_{k=0}^{\infty} \beta_{1}^{k} x^{k}=\frac{1}{1-\beta_{1} x}
$$

in power $m$ for $x:\left|\beta_{1} x\right|<1$ where (13) was used. Result can be written in

Lemma 3:

$$
z_{k, k}^{(m)}=\left.\frac{1}{k !} \partial_{x}^{k} \frac{1}{\left(1-\beta_{1} x\right)^{m}}\right|_{x=0}=\frac{m \cdot \ldots \cdot(m+k-1)}{k !} \beta_{1}^{k}=C_{m+k-1}^{k} \beta_{1}^{k} .
$$

\subsection{About $z_{k, k}^{-1}$ and $z_{k+1, k}^{-1}$ for $k>0$}

By definition $Z$ and $Z^{-1}$ functions satisfy the equality $Z Z^{-1}=Z^{-1} Z=1$. Using (7), (8) and the arbitrariness of the coupling constant $\alpha_{r}$ and parameter of regularization $\varepsilon$ after some calculation it is easy to find the system of recurrent equations for coefficients of $Z^{-1}$ :

Lemma 4: $z_{k+n, k}^{-1}$ for $k \geqslant 0$ and $n \geqslant 0$ satisfy the equalities

$$
z_{0,0} z_{0,0}^{-1}=1, \sum_{k=0}^{j} \sum_{p=0}^{n} z_{n-p+k, k} z_{p+j-k, j-k}^{-1}=0, j>0, n \geqslant 0 .
$$

Answers for $z_{k, k}^{-1}$ and $z_{k+1, k}^{-1}$ can be formulated in two lemmas:

\section{Lemma 5:}

$$
z_{0,0}^{-1}=1, z_{1,1}^{-1}=-\beta_{1}, z_{j, j}^{-1}=0, j>1 .
$$

Proof: To solve the system of equations (16) for $n=0$

$$
\sum_{k=0}^{j} z_{k, k} z_{j-k, j-k}^{-1}=0, j>0
$$

with initial condition $z_{0,0} z_{0,0}^{-1}=1$ it is necessary to use (13) and ansatz for $z_{j, j}^{-1}$ in the form $z_{j, j}^{-1}=\beta_{1}^{j} a_{j}$.

\section{Lemma 6:}

$$
z_{j+1, j}^{-1}=-\frac{\beta_{2} \beta_{1}^{j-1}}{j(j+1)}, j>0 .
$$

Proof: To solve the system of equations (16) for $n=1$

$$
z_{j+1, j}-z_{j, j-1} \beta_{1}+\sum_{k=0}^{j} z_{k, k} z_{j-k+1, j-k}^{-1}=0, j>0
$$

with initial condition $z_{1,0}^{-1}=0$ it is necessary to use (13) and (14), ansatz for $z_{j+1, j}^{-1}$ in the form $z_{j+1, j}^{-1}=\beta_{2} \beta_{1}^{j-1} a_{j}$ and subtraction of $j$ and $j+1$ equalities

$$
\frac{j}{j+1}+\sum_{k=0}^{j} a_{j-k}=0, j>0 \text {. }
$$




\section{About coefficients of effective action}

\subsection{Relations between coefficients of effective action}

As noted above formula for effective action for the Yang-Mills theory in four-dimensional space-time has the form (1) and after dimensional regularization $W(B, \alpha)$ becomes dependent on parameters of regularization $\mu$ and $\varepsilon$ in such view

$$
W_{r e g}^{\mu, \varepsilon}(B, \alpha)=\frac{1}{\alpha \mu^{-\epsilon}} W_{-1}+\frac{1}{\epsilon} W_{0,1}+\sum_{j=0}^{\infty} \sum_{k=j}^{\infty} \frac{1}{\epsilon^{j}} W_{k, j}\left(\alpha \mu^{-\epsilon}\right)^{k}+o(1),
$$

where coefficients depend on background field $B_{\mu}(x)$. Renormalization means substitution $\alpha \rightarrow \alpha(\varepsilon)$ where $\alpha(\varepsilon)$, the bare coupling constant, eliminates divergences in the considered object and satisfies the equality (9). At the same time renormalized coupling constant $\alpha_{r}$ satisfies the Gell-Mann-Low equation (10). After substitution (7) and (9) in (19) and using results (13), (15) and (17) this can be verified that the renormalized effective action has the form

$$
W_{r e n}^{\mu}\left(B, \alpha_{r}\right)=\frac{1}{\alpha_{r}} W_{-1}+\sum_{k=0}^{\infty} \alpha_{r}^{k} W_{k, 0}
$$

and coefficients of effective action (19) and $Z$ - function should satisfy the relations from

Lemma 7:

$$
\begin{gathered}
z_{1,1}^{(-1)} W_{-1}+W_{0,1}=0, \\
z_{p+m+1, m}^{(-1)} W_{-1}+\sum_{i=0}^{m} \sum_{n=0}^{p} W_{n+m-i, m-i} z_{p-n+i, i}^{(n+m-i)}=0, m \geqslant 1, p \geqslant 0 .
\end{gathered}
$$

\section{2 $W_{k, k}$ coefficients for $k>0$}

To find the answer for coefficients $W_{k, k}$ it is necessary to use the equality (22) with $p=0$

$$
z_{m+1, m}^{(-1)} W_{-1}+\sum_{i=0}^{m} W_{m-i, m-i} z_{i, i}^{(m-i)}=0, m \geqslant 1 .
$$

Then using results (13), (15), (18) and the relation $z_{i, i}^{(0)}=\delta_{0 i}$ the system (23) can be rewritten in such form

$$
-\frac{\beta_{2} \beta_{1}^{m-1}}{m(m+1)} W_{-1}+\sum_{i=0}^{m-1} W_{m-i, m-i} \beta_{1}^{i} C_{m-1}^{i}=0, m \geqslant 1,
$$

which gives the answer

Theorem:

$$
W_{k, k}=W_{-1} \frac{(-1)^{k-1} \beta_{2} \beta_{1}^{k-1}}{k+1} .
$$

Proof: It follows from (23) that it is necessary to find the solution in the form $W_{k, k}=$ $W_{-1} \beta_{2} \beta_{1}^{k-1} a_{k}$. In such way achieving the goal goes from (30) and rewritten LHS of (23) in the form

$$
-\frac{1}{m(m+1)}+\sum_{i=1}^{m} a_{i} C_{m-1}^{i-1}=\frac{1}{m+1}-\frac{1}{m}-\sum_{i=0}^{m} a_{i} C_{m}^{i}+\sum_{i=0}^{m-1} a_{i} C_{m-1}^{i} .
$$




\section{Appendix}

1) Binomial coefficients $C_{n}^{k}$ are defined by the formula

$$
C_{n}^{k}=\frac{n !}{k !(n-k) !}, k, n \in \mathbb{N} \cup\{0\}: n \geqslant k \geqslant 0
$$

and have properties

$$
C_{n}^{k}=C_{n}^{n-k}, C_{n}^{k}=C_{n-1}^{k}+C_{n-1}^{k-1} .
$$

2) Harmonic numbers $H_{n}$ are defined by the formula

$$
H_{n}=\sum_{k=1}^{n} \frac{1}{k}, n \in \mathbb{N}
$$

and have properties

$$
H_{n}=H_{n-1}+\frac{1}{n}, H_{n}=\sum_{k=1}^{n} \frac{(-1)^{k-1}}{k} C_{n}^{k} .
$$

Combining formulas (27) and (29) it is easy to write

$$
H_{n+1}-H_{n}=\sum_{k=0}^{n} \frac{(-1)^{k}}{k+1} C_{n}^{k} .
$$

In addition these numbers have asymptotic behavior

$$
H_{n}=\ln n+\gamma+O\left(\frac{1}{n}\right)
$$

when $n \rightarrow+\infty$, where $\gamma$ is the Euler's constant.

\section{Acknowledgments}

This research is supported by a grant from the Russian Foundation for Basic Research (Project No. 17-01-00283-A).

\section{References}

[1] D.J. Gross, F. Wilczek, Physical Review Letters, 30 (26), 1343-1346 (1973)

[2] H.D. Politzer, Physical Review Letters, 30 (26), 1346-1349 (1973)

[3] S. Coleman, E. Weinberg, Phys. Rev. D, 7, 1888 (1973)

[4] L. D. Faddeev and A. A. Slavnov, Gauge Fields: Introduction To Quantum Theory, Front. Phys. 50 (1980)

[5] L. D. Faddeev, Theoret. and Math. Phys., 148:1, 986-994 (2006)

[6] L. D. Faddeev, Mass in Quantum Yang-Mills Theory: Comment on a Clay Millenium problem, arXiv:0911.1013 [math-ph] (2009)

[7] I. Y. Arefeva, L. D. Faddeev and A. A. Slavnov, Theor. Math. Phys., 21:3, 1165-1172 (1975)

[8] L. D. Faddeev, Int. J. Mod. Phys. A, 31, 1630001 (2016)

[9] A. V. Ivanov, EPJ Web of Conferences, 158, 07004 (2017)

[10] S. E. Derkachev, A. V. Ivanov, L. D. Faddeev, Theoret. and Math. Phys., 192:2, 1134-1140 (2017) 
[11] G. 't Hooft and M. Veltman, Nucl.Phys. B, 44, 189-213 (1972)

[12] C. G. Bollini and J. J. Giambiaggi, Phys. Lett. B, 40, 566-568 (1972)

[13] J. Zinn-Justin, Quantum Field Theory and Critical Phenomena, Clarendon Press (2002)

[14] S. Weinberg, The Quantum Theory of Fields, Cambridge University Press (1999)

[15] J. Collins, Renormalization: An Introduction to Renormalization, the Renormalization Group and the Operator-Product Expansion, Cambridge University Press (1984)

[16] A. V. Ivanov, Zap. Nauchn. Sem. POMI, 465, POMI, St. Petersburg, 147-156 (2017)

[17] David J. Gross, Applications of the Renormalization Group to High-Energy Physics, Methods in Field Theory: Les Houches Session XXVIII, 141-250 (1981) 